Laue results, which enabled the preparation of oriented samples.

The laser resonator was a three-mirror folded cavity configuration to allow compensation of a slight astigmatism introduced by the laser crystal. The 2.2-mmlong laser crystal was placed at a Brewster's angle close to the plane highreflectivity mirror and was mounted on a copper heat sink without any cooling system. The Tm: $\mathrm{BaY}_{2} \mathrm{~F}_{8}$ was longitudinally pumped by a GaAlAs laser diode at a wavelength of $\sim 780 \mathrm{~nm}$ with a maximum cW output power of $3 \mathrm{~W}$. Tuning of the laser wavelength was accomplished by adjustment of an intracavity quartz plate acting as a birefringent filter. The freerunning laser showed cw oscillation over a wide range of wavelengths that depended on the specific configuration. Increasing the output coupling increased the output power, but decreased the wavelength tuning range. The lower-wavelength end of the tuning range (i.e., $1850 \mathrm{~nm}$ ) was limited by the bandwidth of the dielectric laser mirrors that were used. The wavelengthshift of up to $2059 \mathrm{~nm}$, compared with the longest emission wavelength observed in the fluorescence spectrum, at $1925 \mathrm{~nm}$, is ascribed to the vibronic behavior of the laser crystal. To the researchers' knowledge, this is the largest shift ever observed for a rare-earth laser.

ROSALÍA SERNA

\section{Nanotube Coupled with Nanodiamond Forms Hybrid Nanomaterial}

The increasing scale of device integration in solid-state technology, coinciding with decreasing structure dimensions, requires the use of nanomaterials that can be engineered to form nanoscale architectures, built from units with desired shapes and properties. Moreover, such materials must survive under severe conditions. In this context, the family of carbon nanostructures is ideally suited for a wide range of innovative applications, and their chemical compatibility makes hybrid nanomaterials that couple nanotubes and nanodiamond an attractive prospect. Recently, M.L. Terranova and colleagues of the University of Rome
"Tor Vergata," M. Rossi of the University of Rome "La Sapienza," and A.S. Barnard of Argonne National Laboratory have produced ordered deposits of tubular nanostructured carbon that couple diamond nanocrystals with single-walled carbon nanotubes.

In a forthcoming issue of Chemistry of Materials (DOI: 10.1021/cm0502018), Terranova, Rossi, and colleagues outlined a systematic method for the growth of these hybrid nanocarbons, involving the reaction of graphitic carbon nanopowders (produced by cw-laser-assisted pyrolysis of hydrocarbon mixtures) with atomic $\mathrm{H}$, in a modified chemical vapor deposition reactor. The carbon nanopowders were carried from a reservoir by Ar streams and homogeneously delivered across the active area of a Si substrate coated by a submicron dispersion of Fe particles. The experimental parameters were outlined, and it was shown that under well-defined conditions, the production of ordered arrays of rigid nanotubes coated by diamond nanocrystals was possible during the same run. The final deposits consisted of vertically aligned, tubular bundles of singlewalled carbon nanotubes up to $15 \mu \mathrm{m}$ long, coated with an outer deposit of wellshaped diamond nanocrystals (see Figure 1), attached to the nanotubes by a reduced portion of their base. To facilitate the investigation of the formation of these materials, the researchers constructed a time-growth sequence by ad hoc experiments performed by changing the duration of the synthesis process in the range of 1-15 $\mathrm{min}$. The samples for each deposition time were characterized, and the growth and evolution of each of the respective nanocarbon phases were confirmed.

The researchers proposed that following nanotube growth, the high relative concentration of atomic $\mathrm{H}$ used in the experiments was instrumental in disrupting locally the C-C $s p^{2}$ network of the nanotube walls, thereby creating localized C-C $s p^{3}$ defects that act as suitable sites for the nucleation of diamond nanocrystals. As soon as the first diamond crystallites were formed, further top-growth of the nanotube was repressed, since further buildup of nano-

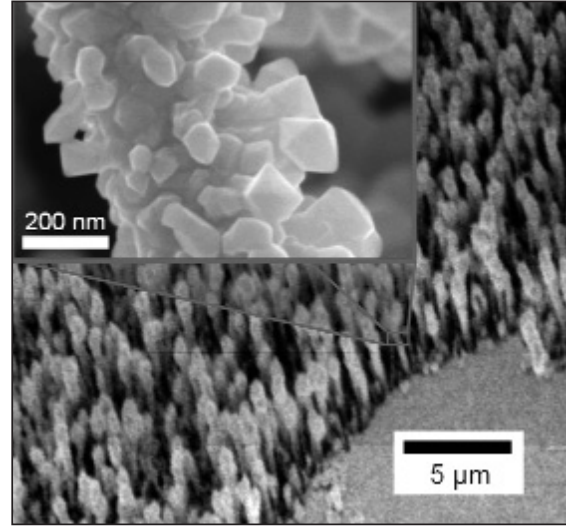

Figure 1. Vertically aligned, tubular bundles of single-walled carbon nanotubes up to $15 \mu \mathrm{m}$ long, coated with an outer deposit of 20-100 nm of nanodiamond, along with a detailed image (inset) showing the faceted shape of the diamond nanocrystals.

tubes was limited by the initial coverage of diamond nanocrystals. Following crystallization, the nanodiamond growth continued, taking advantage of the surface supersaturation and localized carbon segregation produced by the etching of some of the nanotubes as well as that of the feeding $C$ nanopowders. The final coating of diamond nanocrystals remained as "hanging objects," due to the curvature of the (nanotube) substrate, with typical sizes ranging from $20 \mathrm{~nm}$ near the base of the nanotubes to $100 \mathrm{~nm}$ at the top.

Considering that diamond is one of the most promising wide-bandgap semiconductors for various electronic devices, with an excellent potential due to its negative electron affinity, the researchers said that these rigid rods may find applications as micro- and nanoscale cold-cathode devices, miniaturized cathode-ray tubes, light-emitting displays, micromechanics, and nanoscale sensing.

For more research news on Materials Science, access the Materials Research Society Web site: www.mrs.org/connections

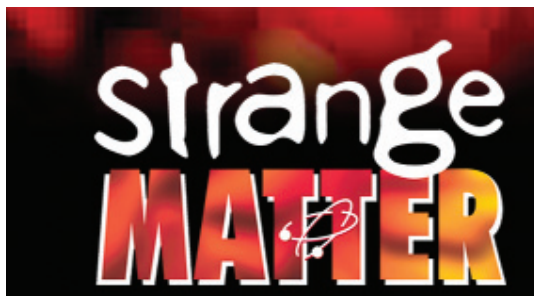

Experience the interactive materials science exhibition:

Cranbrook Institute of Science, Bloomfield Hills, MI June 4 - September 5, 2005

Dallas Museum of Natural History Dallas, TX May 21 - Sept. 4, 2005

To volunteer for activities with the exhibition, contact Amy Moll

Community Resources Coordinator, amoll@boisestate.edu

Strange Matter is presented by the Materials Research Society. This exhibition and its tour are made possible by the generous support of the National Science Foundation, Alcan Inc., Dow, Ford Motor Company Fund, Intel Innovation In Education, and the $3 \mathrm{M}$ Foundation.

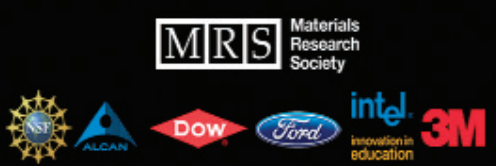

